\title{
Scalar perceptions of distance for a monocularly determined depth interval
}

\author{
DONALD H. MERSHON, MARTIN G. VONCANNON, and WILLIAM R. WINDES \\ North Carolina State University, Raleigh, North Carolina 27607
}

\begin{abstract}
Even in the absence of the usual cues to egocentric distance, a single visual object tends to be seen at an "egocentric reference distance" as a combined result of a perceptual tendency and the residual oculomotor cues. It has previously been shown that two objects, separated in binocular depth, tend to be seen with the far object located near this reference distance and the near object displaced toward the observer. The present research demonstrates that a similar perceptual positioning of a depth configuration will also occur, if the depth interval is established through multiple monocular cues, rather than stereoscopically.
\end{abstract}

Scalar perceptions of egocentric distance are possible even for a single object under reduced viewing conditions in which the majority of cues to distance are absent. The basis for such perceptions has been attributed to the tendency for an isolated object to appear at a relatively near distance (about $2 \mathrm{~m}$ ), independent of the available cues. This phenomenon has been termed the specific distance tendency (SDT) by Gogel (1969). Although the SDT is conceptually independent of the oculomotor cues to distance (i.e., the accommodation and convergence of the eyes), some state of such oculomotor cues will exist in any visual situation and may modify the perceptual outcome. Gogel (1972) has used the combined processes of SDT and oculomotor cues as the basis for what he calls the "egocentric reference distance" and as an explanation for the occurrence of scalar perceptions of extent. A single object presented under reduced viewing conditions, therefore, would be expected to appear at approximately this reference distance. The present research, however, is directed to the question of how a configuration of two objects, separated in depth, is perceptually positioned with respect to this egocentric reference distance.

This question has been investigated by Gogel (1972) and by Mershon and Lembo (in press). In all of these studies, the depth interval was produced with the binocular disparity cue. Although the binocular cue has a practical advantage in that it can provide a powerful relational cue without involving strong direct cues to egocentric distance, the exclusive use of that cue limits the generality of the conclusions. Thus, the conclusion from these studies that the farther object in a depth configuration tends to be perceptually positioned near the egocentric reference distance (defined as the average of the median reported distances of the objects when seen singly) and the nearer object is displaced inward from that distance may be true only for depth intervals established stereoscopically. Gogel (1972), however, has suggested that any relationally determined depth interval should result in the same perceptual positioning. The only requirement for conducting a test of this prediction is that the objects in the depth configuration not yield significantly different reports of perceived distance when seen alone (for separate groups of observers). If they were seen at significantly different distances alone, it would suggest the presence of strong direct cues to the egocentric distances of the objects from the observer. Hence, the individual presentation reports could not be combined into an estimate of a single egocentric reference distance.

Since any given monocular cue may not produce as unambiguous a depth interval as binocular disparity, the present experiment attempted to produce a clear monocular depth separation through the simultaneous use of several monocular cues. Thus, relative size, relative accommodation, and movement parallax were all available as cues to depth. In addition, the objects were positioned at different heights in the visual field, in order to take advantage of that possible factor (cf. Gruber, King, \& Link, 1963). These variations were combined, in order to insure a clear depth separation of the two objects in the configuration, despite the probable introduction of some direct cues to egocentric distance. The presence of such absolute cues was expected to show up for the individual presentations of the objects. If such unavoidable cues were a serious factor in determining the egocentric positions of the objects, then a statistically significant difference in the perceived distance of the single objects should occur. If the distance reports for those presentations did not differ significantly, then it would be possible to proceed with the analysis for the two-object configuration.

\section{METHOD}

\section{Apparatus}

The stimuli consisted of two rectangular cut-outs positioned at different distances in front of a uniformly luminous surface $(.21 \mathrm{~mL})$. The rectangles were centered (one above the other) in the observer's median plane. The top edge of the higher ("far") rectangle was positioned at the observer's eye level and at a distance of $212.0 \mathrm{~cm}$. The top edge of the lower ("near") rectangle was positioned $2.72 \mathrm{deg}$ below eye level and at a distance of $158.0 \mathrm{~cm}$ from the observer. The physical dimensions of the two rectangles were such that the far rectangle was 
Table 1

Medians. Semi-Interquartile Ranges, and Geometrical Means of Perceived Distance $\left(D^{\prime}\right)$ for Single and Simultaneous Presentations of the Two Monocular Rectangles

\begin{tabular}{|c|c|c|c|c|c|}
\hline & \multicolumn{3}{|c|}{ Single Presentations } & \multicolumn{2}{|c|}{$\begin{array}{l}\text { Simultaneous } \\
\text { Presentation }\end{array}$} \\
\hline & \multicolumn{2}{|c|}{ Rectangle } & Average & \multicolumn{2}{|c|}{ Rectangle } \\
\hline Mdn & 137.2 & 304.8 & 221.0 & 91.4 & 182.9 \\
\hline Semi-IQR & 121.9 & 274.3 & & 116.1 & 235.0 \\
\hline G.II & 154.8 & 237.4 & 196.1 & 95.1 & 192.6 \\
\hline
\end{tabular}

- iote-All values are comerted to centimeters.

1.38 deg wide $x .88$ deg high and the rear rectangle was $2.72 \mathrm{deg}$ $\times 1.70$ deg high. Cardboard flaps allowed presentation of either rectangle alone or both rectangles simultaneously. Care was taken that nothing other than the rectangle(s) was visible to the observer at any time during the experiment, with the remainder of the visual field kept totally dark. Although only one light source was used, each rectangle appeared at the distance of its respective cut-out (cf. Lichten \& Lurie, 1950).

All observations were made from inside a dark booth which contained an adjustable chin rest. a communications system, and a shutter for occluding all stimuli prior to the presentation.

\section{Observers}

The observers were 60 students from an introductory course in psychology serving to fulfill course requirements. Each had a visual acuity of at least 20/30 in his/her left eye (corrected if necessary). All were naive as to the purpose of the experiment.

\section{Procedure}

The observers were divided randomly into three groups. Twenty observers were presented the near rectangle alone. Twenty observers were presented the far rectangle alone. Twenty observers were presented both rectangles simultaneously. Presentation of the three conditions was in randomly ordered blocks.

Each observer was screened for visual acuity before being taken into the observation booth and given instructions about the task. An eye patch was placed over the observer's right eye. One minute of dark adaptation then preceded the presentation. During this delay, white noise was delivered to the observer's earphones, in order to mask extraneous noises from the laboratory or hallway.

Monocular vision was used throughout. Observers were instructed that small rocking motions of the head might allow clearer perceptions for their judgments. For observers shown a single rectangle, the task was to report verbally ("in feet or inches or in some combination of feet and inches") the apparent distance to the rectangle. For observers shown the two rectangles simultaneously, the task was to indicate, first, whether or not there was a perceived depth separation of the rectangles, and, then. to report verbally the apparent distance of each of the rectangles. The order of report for the two rectangles (in the multiple-presentation condition) was counterbalanced across observers. If an observer initially reported both rectangles to appear at the same distance, that observer was replaced. (A total of four observers beyond the indicated 20 failed to perceive a depth interval and were rejected from the sample.) Each observer served in only one condition and received only one presentation.

\section{RESULTS AND DISCUSSION}

The results of the experiment are shown in Table 1. The original reports of perceived distance $\left(D^{\prime}\right)$, expressed in feet and inches, have all been converted to centimeters. Since some of the distributions are skewed and/or contain a few extreme values (cf. Mershon \& Lembo, in press. for a discussion of this problem with the verbal report procedure). medians rather than arith metic means best represent these data. Geometrical mean $(\mathrm{GM})$ values. which are also less sensitive to skew. have been presented for additional comparison. The difference in perceived distance for the near rectangle alone and the far rectangle alone, although somewhat large, was not significant (Mann-Whitney $L$ test. $\mathrm{U}=159, \mathrm{p}>.10)$. The average of these median values from the single presentations of the rectangles can thus be used as an estimate for the egocentric reference distance. This value of $221.0 \mathrm{~cm}$ can then be applied to the $D^{\prime}$ reports for the simultaneous presentation. in order to answer the original question about the positioning of a monocular configuration.

The median $\mathrm{D}^{\prime}$ values obtained from the simultaneous presentation of the rectangles were 91.4 and $182.9 \mathrm{~cm}$ for the near and far rectangles, respectively. Each of these values was less than the median perceived distance of the corresponding rectangle in the single presentations and also less than the egocentric reference distance. Neither comparison of a rectangle alone with the same rectangle in the simultaneous presentation was significant by the Mann-Whitney $\mathrm{U}$ test, although the comparison for the near rectangle approached significance $[\mathrm{U}=142, \mathrm{z}($ corrected for ties $)=1.58, \mathrm{p}$ (one-tailed $)<$ .06]. Comparison of the reports from the simultaneous presentations with the estimated value of the egocentric reference distance showed no significant displacement for the far rectangle. The near rectangle was, however, significantly forward from the egocentric reference distance ( $p=.02$ by sign test).

Thus, it seems that a pair of luminous rectangles separated by a monocular depth interval is positioned in apparent distance such that the egocentric reference distance is very close to. but farther from. the observer than is the farther rectangle in the pair. The nearer rectangle is displaced significantly toward the observer from that reference distance. (It may be noted that the geometrical mean data confirm this same pattern of results, without quite as large a difference between the single object presentations as the medians.) These results are consistent with the general findings of both Gogel (1972) and Mershon and Lembo (in press). They demonstrate, in addition, that a depth interval need not be generated by the stereoscopic cue and viewing need not be binocular in order for the phenomenon to occur.

\section{REFERENCES}

Gogel. W. C. The sensing of retinal size. Vision Research 1969. 9. $10^{-9} 9 \cdot 1094$.

Gogel. W. C. Scalar perceptions with binocular cues of distance. American Journal of Psychology. 19-2. 85. $4^{-7^{--4}} \cdot 9^{-}$.

Grtber. H. E.. King. W.. \& Link. S. Moon illusion: An event in imaginary space. Science. 1963.139.750-752.

Lichtex. W.. \& Lirie. S. A new technique for the study of perceived size. American Joumal of Psychology. 19\$0. 63. 280-282.

Mershos. D. H.. \& Lembo. V. L. Scalar perceptions of distance in simple binocular configurations. American Journal of Psychology. in press.

(Received for publication June 21. 1976.) 